\title{
Corner Flow of Power Law Fluids
}

\author{
Henriksen, P.; Hassager, Ole
}

Published in:

Journal of Rheology

Link to article, DOI:

10.1122/1.550039

Publication date:

1989

\section{Document Version}

Publisher's PDF, also known as Version of record

Link back to DTU Orbit

Citation (APA):

Henriksen, P., \& Hassager, O. (1989). Corner Flow of Power Law Fluids. Journal of Rheology, 33(6), 865-879. https://doi.org/10.1122/1.550039

\section{General rights}

Copyright and moral rights for the publications made accessible in the public portal are retained by the authors and/or other copyright owners and it is a condition of accessing publications that users recognise and abide by the legal requirements associated with these rights.

- Users may download and print one copy of any publication from the public portal for the purpose of private study or research.

- You may not further distribute the material or use it for any profit-making activity or commercial gain

- You may freely distribute the URL identifying the publication in the public portal

If you believe that this document breaches copyright please contact us providing details, and we will remove access to the work immediately and investigate your claim. 


\title{
Corner Flow of Power Law Fluids
}

\author{
P. HENRIKSEN and O. HASSAGER, Instituttet for \\ Kemiteknik, Danmarks tekniske Højskole, \\ 2800 Lyngby, Denmark
}

\section{Synopsis}

A local analysis of the flow of power law fluids near corners is performed. The equation for the stream function is shown to allow separated solutions in plane polar coordinates. The radial behavior is shown to be algebraic and results are given for the exponent for different values of corner angle and power law exponent. In addition, the critical angle for the onset of an eddy structure is found as function of the power law exponent.

\section{INTRODUCTION}

We consider the local plane creeping two-dimensional flow near two plane boundaries meeting at an angle $2 \alpha$ at a sharp corner as shown in Figure 1. The velocity vanishes on both boundaries. The flow near the corner is induced by some motion far from the corner. The equation for the stream function was shown by Dean and Montagnon ${ }^{1}$ to have separated solutions of the form

$$
\psi=r^{\lambda} f(\theta)
$$

for Newtonian fluids. When the angle $2 \alpha$ is smaller than a critical value $2 \alpha_{c}$, the exponent $\lambda$ is complex, and the flow near the corner was shown by Moffatt to consist of an infinite sequence of eddies. ${ }^{2}$ An asymptotic solution for the steady flow near a stationary contact line at a plane boundary for two immiscible incompressible Newtonian liquids was presented recently by Proudman and Asadullah. ${ }^{3}$

In a related development, Hutchinson ${ }^{4}$ analyzed the singular behavior at the end of a tensile crack in a hardening material. Hutchinson's result may be adopted ${ }^{5}$ for the flow of a power law fluid around a corner of angle $2 \alpha=2 \pi$. The general method was further used by Fenner ${ }^{6}$ to analyze the flow of power law fluids near corners of angles $2 \alpha<\pi$. The behavior of viscoelastic fluids near sharp corners is a matter of considerable current interest ${ }^{7-9}$ but remains largely an unresolved problem.

(c) 1989 by The Society of Rheology, Inc. Published by John Wiley \& Sons, Inc. Journal of Rheology, 33(6), 865-879 (1989) CCC 0148-6055/89/060865-15\$04.00 


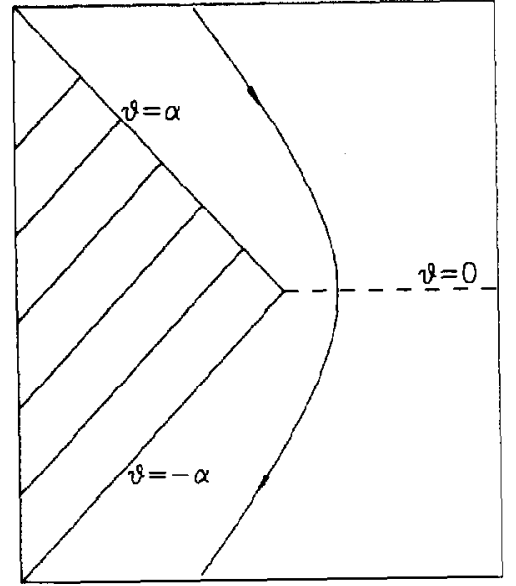

(a)

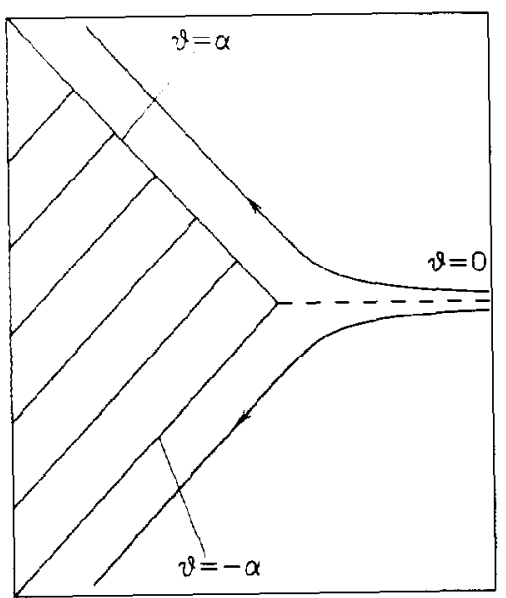

(b)

Fig. 1. Streamlines for three different kinds of flow situations investigated in this work: (a) antisymmetrical flow, $\alpha=15 \pi / 20$, (b) symmetrical flow, $\alpha=$ $15 \pi / 20$, and (c) symmetrical flow with a free surface $\alpha=30 \pi / 20$. The streamlines are only shown on a qualitative basis.

\section{ANALYSIS}

The analysis applies to incompressible generalized Newtonian fluids, that is fluids for which the stress tensor $\tau$ is given by

$$
\tau=-\eta \dot{\gamma}
$$




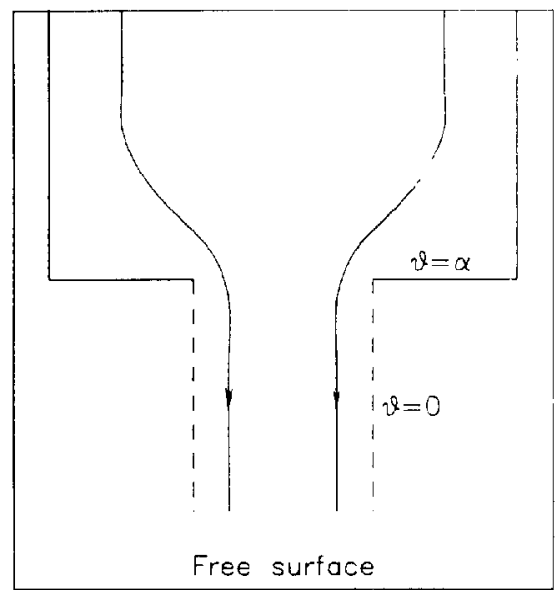

(c)

(Fig. 1 continued)

where the rate-of-strain tensor is

$$
\dot{\gamma}=(\boldsymbol{\nabla} \boldsymbol{v})+(\boldsymbol{\nabla} \boldsymbol{\nu})^{+}
$$

The viscosity function $\eta$ in Eq. (2) is a function of the scalar invariants of $\dot{\gamma}$. It is customary to consider only the magnitude of $\dot{\gamma}$ which we define as

$$
\dot{\gamma}=\sqrt{\frac{1}{2} \dot{\gamma}: \dot{\gamma}}
$$

It is assumed also that the viscosity function (at least at the conditions near the corner) is described by a power law

$$
\eta=m \dot{\gamma}^{n-1}
$$

where $m$ and $n$ are the power law parameters. The neglect of dependence of $\eta$ on $\operatorname{tr} \dot{\gamma}$ is legitimate since $\operatorname{tr} \dot{\gamma}=0$ for incompressible flow. Furthermore, the neglect of dependence on det $\dot{\gamma}$ is legitimate since det $\dot{\gamma}=0$ for plane flow. We assume that the flow near the corner is locally a plane flow, not only for those flow situations that are plane flow globally, but also for those flow situations that are axisymmetric globally. We define components of the fluid velocity in plane polar coordinates $\nu_{r}(r, \theta)$ and $\nu_{\theta}(r, \theta)$ related to the stream function $\psi(r, \theta)$ as follows

$$
\nu_{r}(r, \theta)=-\frac{1}{r} \frac{\partial}{\partial \theta} \psi(r, \theta)
$$




$$
\nu_{\theta}(r, \theta)=\frac{\partial}{\partial r} \psi(r, \theta)
$$

Then we assume that the stream function for the flow near the corner may be expanded in a series of the following form

$$
\psi(r, \theta)=\sum_{k=1}^{\infty} \operatorname{Re}\left\{A_{k} r^{\lambda_{k}} f_{k}(\theta)\right\}
$$

where

$$
\lambda_{0}<\operatorname{Re}\left\{\lambda_{1}\right\}<\operatorname{Re}\left\{\lambda_{2}\right\} \cdots
$$

Here $\lambda_{0}=\lambda_{0}(n)$ (= 1 for Newtonian fluids) is determined by the following three restrictions: (i) The inertial forces must be negligible compared with the viscous forces. The order of magnitude of the inertial acceleration is $O(\boldsymbol{v} \cdot \nabla \boldsymbol{\nu})=\left(r^{2 \lambda-3}\right)$, while the order of magnitude of the viscous forces is $O(\nabla \cdot \tau)=\left(r^{n(\lambda-2)-1}\right)$. Hence the inertial terms become negligible for sufficiently small $r$ provided

$$
2 \frac{n-1}{n-2}<\lambda \quad(0<n<2)
$$

For $n=1$ this gives $\lambda>0$ in agreement with the condition of Moffatt. $^{2}$ (ii) In addition, the rate of energy dissipation must be finite over a small volume of fluid near the corner. The order of the energy dissipation is $O(\tau: \nabla \nu)=\left(r^{(n+1)(\lambda-2)}\right)$. Hence for the integral $\int(\tau: \nabla v) r d r d \theta$ to exist we must require

$$
\lambda>\frac{2 n}{n+1}
$$

in agreement with Hutchinson. ${ }^{4}$ This condition also ensures the integrability of the variational integral for the generalized Newtonian fluid. ${ }^{10}$ (iii) Finally, we must require that the pressure or any other part of the stress imparts a finite force on the corner. The order of magnitude of the pressure is $O(p)=\left(r^{n(\lambda-2)}\right)$. Hence for the integral $\int p d r d z$ to exist we must require

$$
\lambda>2-\frac{1}{n}
$$

We note that there is a logical distinction between the first condition (which insures that the solution is asymptotically correct as $r \rightarrow 0$ ) and the two latter conditions (which are postulated on physical grounds). Nonetheless the conditions are combined to $\lambda_{0}(n)$ in Figure 2. 
To arrive at a local analysis we introduce the expression for the velocity components in Eqs. (6)-(8) into the expression for $\dot{\gamma}$ in Eq. (4) formulated in plane polar coordinates. The result of the double contraction is a sum of terms with a factor $r$ raised to powers $\left(2 \lambda_{1}-4\right),\left(\lambda_{1}+\lambda_{2}-4\right),\left(2 \lambda_{2}-4\right),\left(\lambda_{1}+\lambda_{3}-4\right), \ldots$ According to Eq. (9), the terms associated with $\lambda_{1}$ alone will dominate when $r \rightarrow 0$. Therefore, in a local analysis we drop all terms except those that arise from $\lambda_{1}$ alone. The result for the magnitude of the rate-of-strain tensor is

$$
\dot{\gamma}=A r^{\lambda-2} \sqrt{\left[2(\lambda-1) f^{\prime}\right]^{2}+\left[\lambda(\lambda-2) f-f^{\prime \prime}\right]^{2}}
$$

Here and in the following we let $\lambda_{1}=\lambda, A_{1}=A$ and $f_{1}(\theta)=f$.

The following explicit expressions for the non-zero stress components $\tau_{r r}, \tau_{\theta \theta}$, and $\tau_{r \theta}$ are obtained from Eqs. (2) and (3) formulated in component form in plane polar coordinates

$$
\begin{aligned}
& \tau_{r r}=2 A(\lambda-1) \eta r^{\lambda-2} f^{\prime}=-\tau_{\theta \theta} \\
& \tau_{r f}=-A \eta r^{\lambda-2}\left[\lambda(\lambda-2) f-f^{\prime \prime}\right]
\end{aligned}
$$

Here again, only terms associated with $\lambda_{1}$ are retained in the analysis. To arrive at a closed equation for $f(\theta)$, one formulates the $r$ and $\theta$ components of the equation of motion for creeping flow. The pressure is eliminated between the two by differentiation and subtraction. Furthermore, $\tau_{\theta \theta}$ may be eliminated with the use of the relation $\tau_{\theta \theta}=-\tau_{r r}$ to arrive at

$$
2 \frac{\partial}{\partial \theta}\left(\frac{\tau_{r r}}{r}+\frac{\partial \tau_{r r}}{\partial r}\right)+\frac{1}{r} \frac{\partial^{2} \tau_{\theta r}}{\partial \theta^{2}}-3 \frac{\partial \tau_{\theta r}}{\partial r}-r \frac{\partial^{2} \tau_{\theta r}}{\partial r^{2}}=0
$$

The closed equation for $f(\theta)$ is now obtained by substituting Eq. (5) and Eqs. (13)-(15) in Eq. (16). In the resulting equation the radial part may be factored out completely (together with $A$ and $m$ ). The result is the following 4th-order ordinary differential equation for $f$ :

$$
\begin{aligned}
& 4(\lambda-1)[(\lambda-2) n+1]\left(\frac{n-1}{2} \Gamma \Gamma^{\prime} f^{\prime}+\Gamma^{2} f^{\prime \prime}\right) \\
& -\frac{n-1}{2}\left(\frac{n-3}{2} \Gamma^{\prime 2}+\Gamma \Gamma^{\prime \prime}\right)\left[\lambda(\lambda-2) f-f^{\prime \prime}\right] \\
& -(n-1) \Gamma \Gamma^{\prime}\left[\lambda(\lambda-2) f^{\prime}-f^{\prime \prime \prime}\right]-\Gamma^{2}\left[\lambda(\lambda-2) f^{\prime \prime}-f^{i v}\right] \\
& \quad+n(\lambda-2)[(\lambda-2) n+2] \Gamma^{2}\left[\lambda(\lambda-2) f-f^{\prime \prime}\right]=0
\end{aligned}
$$

where

$$
\Gamma=\left[2(\lambda-1) f^{\prime}\right]^{2}+\left[\lambda(\lambda-2) f-f^{\prime \prime}\right]^{2}
$$


We now consider the question of boundary conditions for the equation in (17) and (18). First we notice that the equation does allow solutions of the form $f(-\theta)=+f(\theta), \theta \in[0, \alpha]$ (corresponding to antisymmetric flow) as well as solutions of the form $f(-\theta)=$ $-f(\theta), \theta \in[0, \alpha]$ (corresponding to symmetric flow). This means that if the disturbance far from the corner is completely antisymmetric we may restrict consideration to the interval $[0, \alpha]$ and use the boundary conditions for the antisymmetric flow [Fig. 1(a)].

$$
\begin{aligned}
f^{\prime}(0)=0, & f^{\prime \prime \prime}(0)=0 \\
f(\alpha)=0, & f^{\prime}(\alpha)=0
\end{aligned}
$$

Similarly, if the disturbance far from the corner is completely symmetric, we may restrict consideration to the interval $[0, \alpha]$ and use the conditions for symmetric flow [Fig. 1(b,c)].

$$
\begin{array}{ll}
f(0)=0, & f^{\prime \prime}(0)=0 \\
f(\alpha)=0, & f^{\prime}(\alpha)=0
\end{array}
$$

The two situations of completely antisymmetric flow and completely symmetric flow are analyzed separately in the following.

For Eqs. (17)-(22) we note the following properties characteristic of eigenvalue problems: First $f(\theta)=0$ is a trivial solution. Second, if $f(\theta)$ is a solution, so is any multiple of $f(\theta)$. That is, for a given $\lambda$ there is either no or an infinite number of solutions for $f(\theta)$. To choose one of these solutions we will take the one that has a prescribed value at $\theta=0 .(f(0)=1$ for antisymmetric flow and $f^{\prime}(0)=1$ for symmetric flow.)

We now follow Hutchinson [4] and solve the eigenvalue problem by a shooting technique. For this purpose we define

$$
\left.\begin{array}{l}
\left(x_{1}, x_{2}\right)=\mathbf{x}=\left(\lambda, f^{\prime \prime}(0)\right), \text { Antisymmetric flow } \\
\left(x_{1}, x_{2}\right)=\mathbf{x}=\left(\lambda, f^{\prime \prime \prime}(0)\right), \text { Symmetric flow }
\end{array}\right\}
$$

and

$$
\left(F_{1}(\mathbf{x}), F_{2}(\mathbf{x})\right)=\left(f(\alpha), f^{\prime}(\alpha)\right)
$$

Our problem is now to find a solution to the nonlinear equations

$$
\left.\begin{array}{l}
F_{1}(\mathbf{x})=0 \\
F_{2}(\mathbf{x})=0
\end{array}\right\}
$$

for given $\alpha$ and $n$. Equation (25) is solved by Newton iteration where $F_{i}(\mathbf{x})$ and the derivatives $\partial F_{i} / \partial x_{j}, i, j=1,2$ are determined by fourth-order Runge-Kutta integration. ( $\partial F_{i} / \partial x_{j}$ is found analytical and integrated together with $f, f^{\prime}, f^{\prime \prime}$, and $f^{\prime \prime \prime}$.) The first 
guess on $\mathbf{x}$ is obtained by extrapolation with Forsythe polynomials [11] from earlier results with different $n$. (And as a start the Moffatt solution ${ }^{2}$ for $n=1$ is used.)

To find the bifurcation curve, that is, the boundary between real and complex eigenvalues, we define

$$
\left.\begin{array}{c}
\left(y_{1}, y_{2}, y_{3}\right)=\mathbf{y}=\left(\lambda, f^{\prime \prime}(0), n\right) \text { Antisymmetric flow } \\
\left(y_{1}, y_{2}, y_{3}\right)=\mathbf{y}=\left(\lambda, f^{\prime \prime \prime}(0), n\right), \text { Symmetric flow }
\end{array}\right\}
$$

At the boundary between real and complex values of $\lambda$ the Jacobian determinant $G_{3}(y)=\operatorname{det}\left(\partial F_{i} / \partial x_{j}\right)$ is 0 , so the system of nonlinear equations for the bifurcation curve is given by

$$
\left.\begin{array}{l}
G_{1}(\mathbf{y})=0 \\
G_{2}(\mathbf{y})=0 \\
G_{3}(\mathbf{y})=0
\end{array}\right\}
$$

Equation (28) is solved in the same way as described for Eq. (25) for different values of $\alpha$ where the first bifurcation point is taken from Moffatt ${ }^{2}$ for $n=1$. To perform the Newton iteration the partial derivatives $\partial^{2} F_{i} / \partial x_{j} \partial x_{k}, i, j, k=1,2$ are needed. They are found analytically and integrated with $f, f^{\prime}, f^{\prime \prime}, f^{\prime \prime \prime}$, and $\partial F_{i} / \partial x_{j}$, $i, j=1,2$.

\section{FINITE-ELEMENT SOLUTIONS}

To obtain an independent check on the procedure, a number of finite-element calculations of the flow near sharp corners were performed. The calculations were performed with a program based on the known ${ }^{10,12}$ variational principle for generalized Newtonian fluids.

Essential boundary conditions may be imposed on some or all of the closed boundary conditions around the flow domain. Where essential boundary conditions are not imposed, the natural boundary conditions are zero traction conditions. The flow domain is divided into triangles, and the velocity field is discretized with 6-node piecewise quadratic shape functions, and the pressure with three-node piecewise linear shape functions. ${ }^{13}$

Let a fixed polar angle $\theta$ define a 'direction' away from the corner with $\theta=0$ along the bisector of the angle. Consider two points $\left(r_{1}, \theta\right)$ and $\left(r_{2}, \theta\right)$ on the same direction, and let $\nu_{r}(1)$ and $\nu_{r}(2)$ be the radial velocity components at the two points. It then follows that if the velocity field is of the form implied by Eq. (4), then $\lambda_{1}$ will be given by 


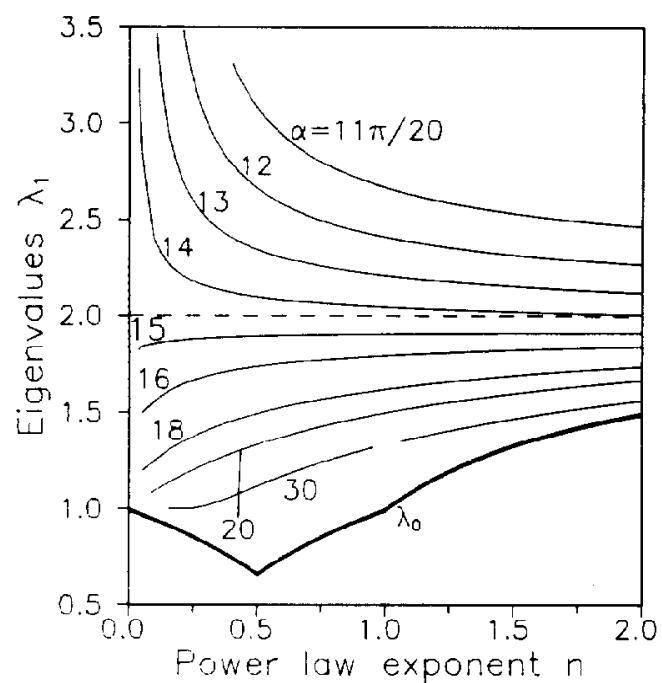

(a)

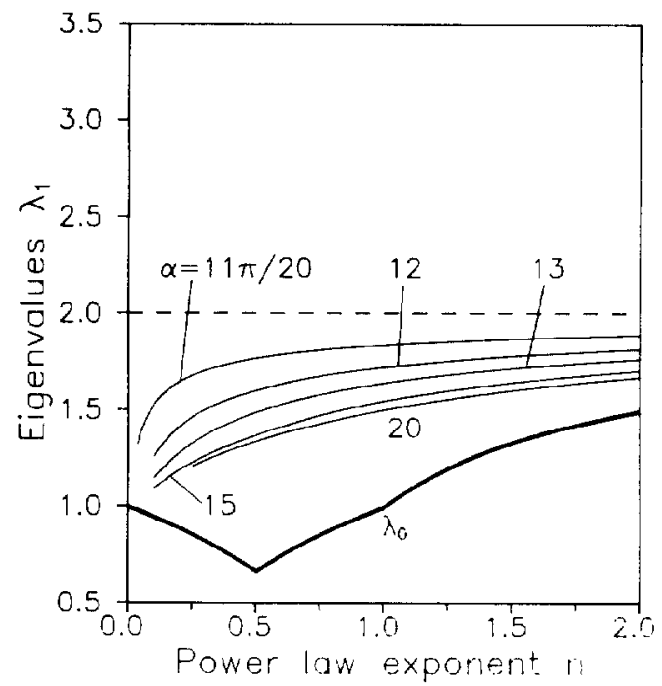

(b)

Fig. 2. Eigenvalues calculated with the Runge-Kutta integration/Newton iteration method for (a) symmetrical flow, $\alpha=11 \pi / 20$ to $16 \pi / 20,18 \pi / 20,20 \pi / 20$, $30 \pi / 20$ and (b) antisymmetrical flow, $\alpha=11 \pi / 20$ to $13 \pi / 20,15 \pi / 20,20 \pi / 20$. There is a pressure singularity at the corner for eigenvalues under the dashed line. The missing part of the $30 \pi / 20$ curve, symmetrical flow, indicates that no eigenvalues could be computed in this range. 


$$
\lambda_{1}=1+\frac{\ln \left[\nu_{r}(2) / \nu_{r}(1)\right]}{\ln \left[r_{2} / r_{1}\right]}
$$

Note that in place of the radial velocity component one could use the angular velocity component $\nu_{\theta}$ or any linear combination of the two, such as $\nu_{x}$ or $\nu_{y}$.

\section{RESULTS FOR EXTERIOR FLOW DOMAINS ( $\alpha>\pi / 2)$.}

Figure 2 and Table I show the values of $\lambda_{1}$ obtained by the corner analysis for $\alpha>\pi / 2$. Note that when $\lambda_{1}<2$, the velocity gradients and stresses are infinite at the corner, and when $\lambda_{1}>2$ the velocity gradients and stresses are zero at the corner. It follows that the stresses are infinite in antisymmetric flow past any corner of total opening angle $2 \alpha>\pi$, irrespective of the value of the power law exponent. For symmetric flow we see that the corner stresses are zero when $\alpha \leq 14 \pi / 20$ and infinite when $\alpha \geq 15 \pi / 20$. It should be noted also that none of the computed eigenvalues violate the lower bound obtained on physical grounds. Finally, we note that for $\alpha-\pi$ we found approximately the same eigenvalues for symmetric and antisymmetric flow.

TABLE I

The eigenvalues $\lambda_{1}$ calculated with the Runge-Kutta integration/Newton iteration method for $\alpha=13 \pi / 20,15 \pi / 20$ and $17 \pi / 20{ }^{\mathrm{a}}$

\begin{tabular}{cccc}
\hline$n$ & $\alpha$ & Symmetrical flow & Antisymmetrical flow \\
\hline 0.50 & $13 \pi / 20$ & 2.3431 & 1.4868 \\
0.75 & - & 2.2556 & 1.57719 \\
1.00 & - & 2.2071 & 1.63673 \\
1.25 & - & 2.1748 & 1.67970 \\
1.50 & - & 2.1511 & 1.71251 \\
& & & \\
0.50 & $15 \pi / 20$ & 1.8984 & 1.37 \\
0.75 & - & 1.9049 & 1.473 \\
1.00 & - & 1.9085 & 1.54448 \\
1.25 & - & 1.9109 & 1.59807 \\
1.50 & - & 1.9127 & 1.63974 \\
& & & \\
0.50 & $17 \pi / 20$ & 1.6050 & 1.24 \\
0.75 & - & 1.6626 & 1.44 \\
1.00 & - & 1.7012 & 1.5088 \\
1.25 & - & 1.7296 & 1.5649 \\
1.50 & - & 1.7517 & 1.6094 \\
\hline
\end{tabular}

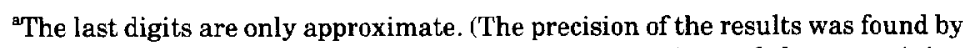
varying the step size $(1 / 10)$ in the Runge-Kutta integration and then examining how many digits in $\lambda_{1}$ were unchanged.) 
For all $\alpha<\pi$ the eigenvalue in antisymmetrical flow is smaller than the eigenvalue in symmetrical flow. This means that any combination of symmetrical and antisymmetrical boundary conditions will result in pure antisymmetrical flow near the corner. To illustrate this statement we performed a finite-element solution of combined symmetrical and antisymmetrical flow past a reentrant corner with $\alpha=15 \pi / 20$. The mesh has a total of 2000 degrees of freedom, and the size of the smallest elements next to the corner is $10^{-3} \times 10^{-3}$ compared to the overall dimension of 1 . The finite-element solution does indeed show pure antisymmetrical flow next to the corner with a $\lambda_{1}$ value that agrees with that determined from the local analysis within $1 \%$. Close to the corner the finite-element solution is inaccurate due to the singularity, while far from the corner there will be a limitation on the local analysis.

The finite-element method may be used to investigate qualitatively the nature of the second eigenfunction as follows. Along any fixed direction $\theta$ away from the corner, the velocity vector based on the dominating eigenfunction $f_{1}$ will have a fixed direction, $\mathbf{a}(\theta)$ say. Hence, if in the same direction $\theta$, we consider the velocity component perpendicular to $\mathbf{a}(\theta)$, we get contributions that are dominated not by $f_{1}$ and $\lambda_{1}$ but by $f_{2}$ and $\lambda_{2}{ }^{9}$. We illustrate this for the combined flow around a corner with $\alpha=3 \pi / 4$ in Table II. Here the contribution from the dominating antisymmetric flow to $\left(\nu_{x}-\nu_{y}\right)$ vanishes along the line $(\theta=0)$. We see that for $n=1$ we pick up the eigenvalue for symmetric flow in the same geometry. This is in agreement with the principle of combination of solutions for a linear equation. For the nonlinear equation when $n \neq 1$ the finite-element solutions clearly point at a second eigenfunction with algebraic behavior in the radial direction. The eigenvalue for this second eigenfunction, however, is not the same one that is found in pure symmetrical flow in the same geometry.

Hutchinson ${ }^{4}$ found that $\lambda_{1}$ was determined "within one tenth of one percent by the simple formula $\lambda_{1}=(2 n+1) /(n+1)$ " at $\alpha=$ $20 \pi / 20$. Since we have determined the precision of our results (by varying the stepsize in the Runge-Kutta integration routine), it was possible to get a validation of the formula. Based on this comparison we guess that Hutchinson's formula is, in fact, exact and not approximate, but we have not been able to prove this.

Figures 3-6 show a number of streamlines for symmetrical and antisymmetrical flow. The streamlines in symmetrical flow can be shown to be nearly independent of $n$, while the stream- 
TABLE II

The term $\lambda_{2}$ Calculated with the Finite-Element Method. ${ }^{a}$

\begin{tabular}{|c|c|c|c|c|c|c|c|}
\hline & \multirow[b]{2}{*}{$r_{1} / \sqrt{2}$} & \multicolumn{6}{|c|}{$r_{2} / \sqrt{2}$} \\
\hline & & 0.001 & 0.003 & 0.006 & 0.010 & 0.030 & 0.060 \\
\hline \multirow{6}{*}{$n=0.50$} & 0.003 & 1.537 & & & & & \\
\hline & 0.006 & 1.564 & 1.608 & & & & \\
\hline & 0.010 & 1.580 & 1.619 & 1.635 & & & \\
\hline & 0.030 & 1.581 & 1.602 & 1.599 & 1.583 & & \\
\hline & 0.060 & 1.570 & 1.583 & 1.575 & 1.558 & 1.519 & \\
\hline & 0.100 & 1.565 & 1.574 & 1.565 & 1.550 & 1.519 & 1.519 \\
\hline \multirow{6}{*}{$n=1.00$} & 0.003 & 1.916 & & & & & \\
\hline & 0.006 & 1.913 & 1.908 & & & & \\
\hline & 0.010 & 1.914 & 1.912 & 1.917 & & & \\
\hline & 0.030 & 1.911 & 1.908 & 1.908 & 1.904 & & \\
\hline & 0.060 & 1.907 & 1.904 & 1.903 & 1.899 & 1.890 & \\
\hline & 0.100 & 1.904 & 1.900 & 1.898 & 1.894 & 1.884 & 1.875 \\
\hline \multirow{6}{*}{$n=1.50$} & 0.003 & 2.070 & & & & & \\
\hline & 0.006 & 2.059 & 2.041 & & & & \\
\hline & 0.010 & 2.054 & 2.039 & 2.036 & & & \\
\hline & 0.030 & 2.047 & 2.036 & 2.035 & 2.034 & & \\
\hline & 0.060 & 2.045 & 2.036 & 2.035 & 2.035 & 2.036 & \\
\hline & 0.100 & 2.043 & 2.034 & 2.033 & 2.032 & 2.030 & 2.022 \\
\hline
\end{tabular}

${ }^{a}$ Combined flow, $\alpha=15 \pi / 20 .\left(\nu_{x}-\nu_{y}\right)$ has been used to calculate $\lambda_{2}$. $\left(r_{1}\right.$ and $r_{2}$ are the distances from the corner in the $(\theta=0)$ direction.)

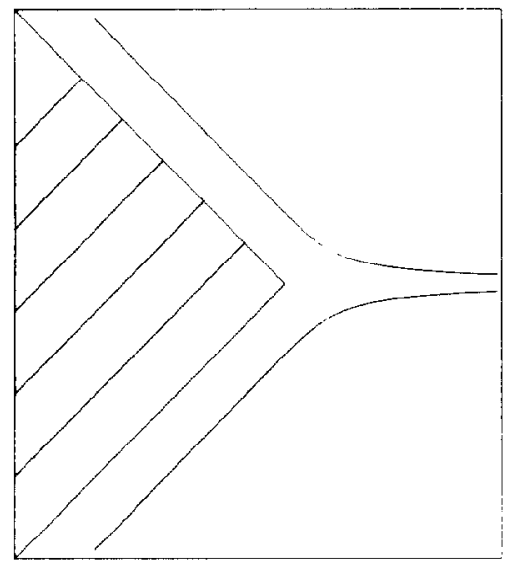

Fig. 3. Streamlines for symmetrical flow, $\alpha=15 \pi / 20$ and $n=1$. 


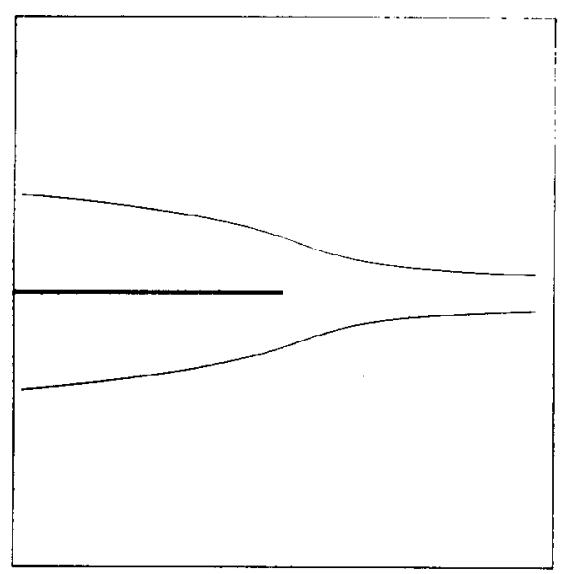

Fig. 4. Streamlines for symmetrical flow, $\alpha=20 \pi / 20$ and $n=1$.

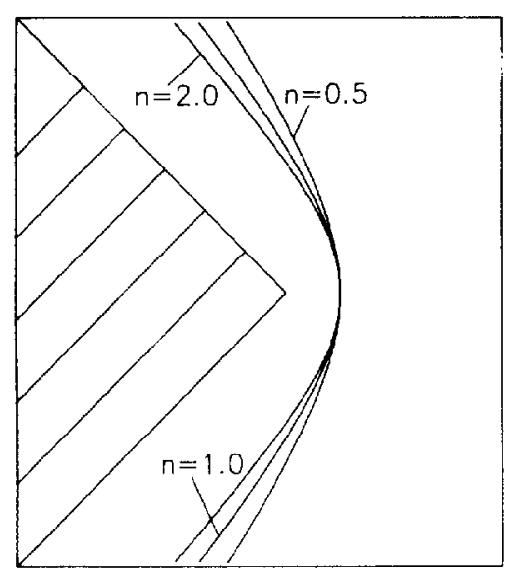

Fig. 5. Streamlines for antisymmetrical flow, $\alpha=15 \pi / 20, n=0.5,1.0$, and 2.0.

lines for antisymmetrical flow do depend significantly on $n$. Note that the "spreading of the streamlines implied by the retardation as the corner is approached" found by Moffatt ${ }^{2}$ is not seen. Quite to the contrary the point of closest approach is located at $\alpha=0$.

We note from Figure 5 that no dividing streamline emanates from the corner. Correspondingly we have checked that there is 


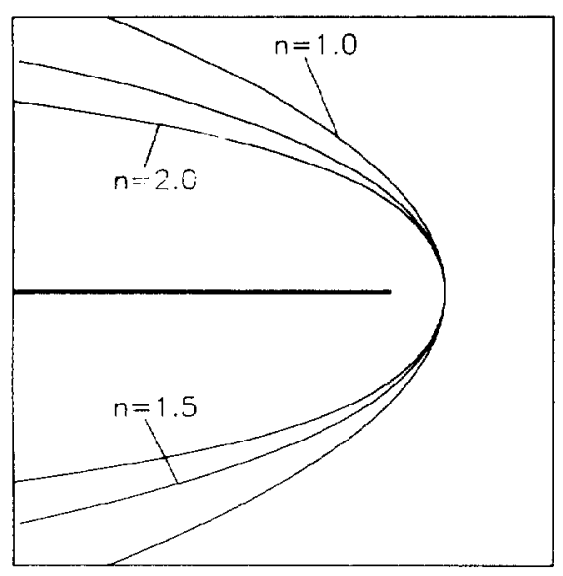

Fig. 6. Streamlines for antisymmetrical flow, $\alpha=20 \pi / 20, n=1.0,1.5$, and 2.0 .

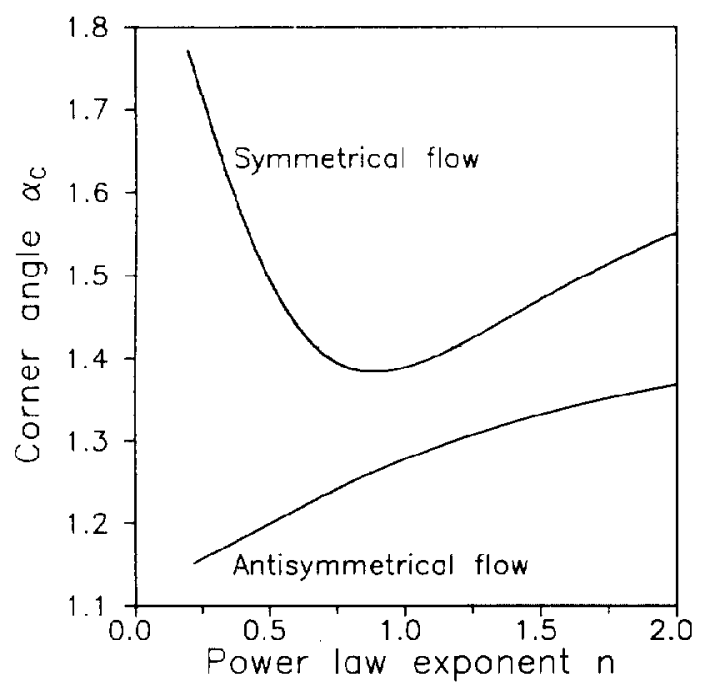

Fig. 7. The critical corner angle $\alpha_{c}$ for onset of an eddy structure as a function of the power law exponent $n$.

no sign change of $f(\theta)$ in the range $-\alpha<\theta<\alpha$ for the dominating eigenfunction. This means that there can be no "lip vortex" at reentrant corners for fluids described by a generalized Newtonian fluid with power law behavior at large deformation rates. 


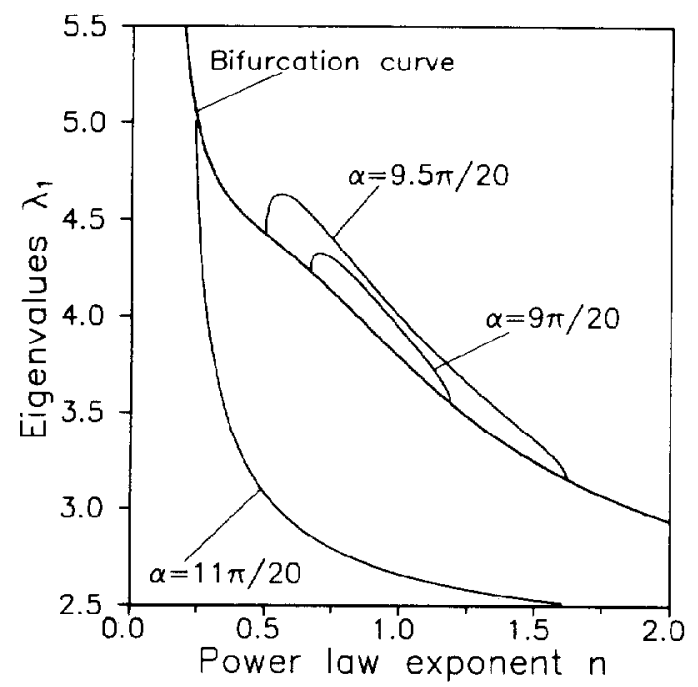

(a)

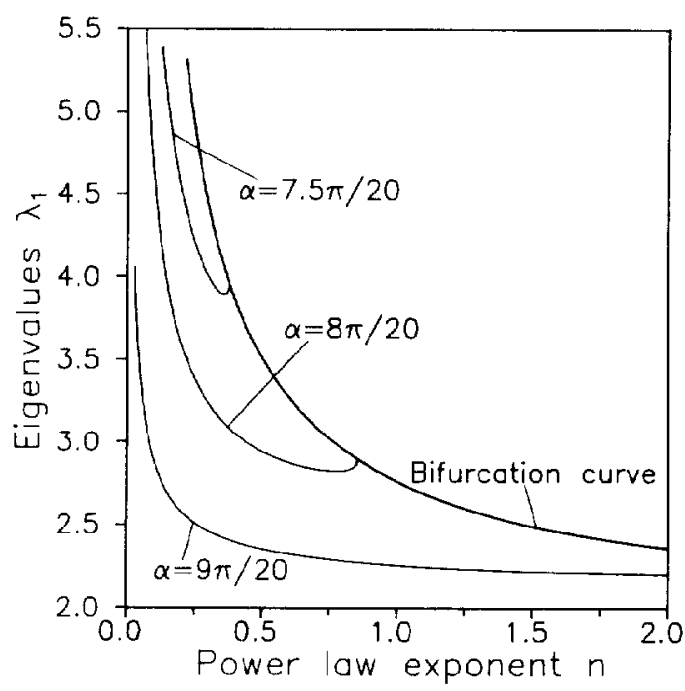

(b)

Fig. 8. Bifurcation and eigenvalue curves where $\alpha$ is the corner angle. (a) Symmetrical flow and (b) antisymmetrical flow.

\section{RESULTS FOR INTERIOR FLOW DOMAINS $(\alpha<\pi / 2)$}

For $\alpha<\pi / 2$ there is the possibility of bifurcation of the solution. The critical corner angle for bifurcation is shown in Figure 7 
for both symmetrical and antisymmetrical flow. The critical corner angle for antisymmetrical flow agrees with the result of Fenner. ${ }^{6}$ Also in Figure 8 we show values of $\lambda_{1}$ for selected angles close to the critical angle together with $\lambda_{1}$ at the critical angle. For $n=1$, the nature of the solution may be investigated also for $\alpha<\alpha_{c}$ and is known to consist of an infinite sequence of eddies. We have not analyzed the structure of the solution for $\alpha<\alpha_{c}$, however we expect that it will describe an infinite sequence of eddies also for $n \neq 1$. We see, moreover, from Figure 8(b) that the value of $\lambda_{1}$ at the critical angle becomes very large for small values of $n$. A large value of $\lambda_{1}$ means that the velocity increases very slowly from zero. We expect, therefore, that power law fluids in interior corners will be practically motionless for $n \ll 1$. While a corner analysis indicates the existence of a sequence of eddies these may be practically impossible to pick up in a finite element simulation. ${ }^{14}$

The work was supported by the Danish Technical Research Council, Grant 5.26.16.00 (Enhanced oil Recovery) and 16-4308.K. We wish to thank the referees and Professor M. L. Michelsen for several useful comments.

\section{References}

1. W. R. Dean, and P. E. Montagnon, Proc. Camb. Phil. Soc., 45, 389 (1949).

2. H. K. Moffatt, J. Fluid Mech., 18, 1 (1964).

3. I. Proudman and M. Asadullah, J. Fluid Mech., 187, 35 (1988).

4. J.W. Hutchinson, J. Mech. Phys. Solids, 16, 13 (1968).

5. R. I. Tanner, Engineering Rheology, Clarendon Press, Oxford, 1985.

6. R. T. Fenner, Int. J. Non Linear Mech., 10, 207 (1975)

7. G. G. Lipscomb, R. Keunings, and M. M. Denn, J. Non-Newtonian Fluid Mech., 24, 85 (1987).

8. R. Keunings, Simulation of viscoelastic fluid flow, in Fundamentals of Computer Modelling for Polymer Processing, C. L. Tucker, ed, Carl Hanser Verlag, 1988.

9. M. Apelian, R. C. Armstrong, and R. A. Brown, J. Non-Newtonian Fluid Mech., 27, 299 (1988).

10. R. B. Bird, R.C. Armstrong, and O. Hassager, Dynamics of Polymeric Liquids, vol. 1, 2 ed., Wiley-Interscience, New York, 1987, eq. 4.3-31.

11. G. E. Forsythe, J. SIAM, 5, 74 (1957).

12. J. Pawlowski, Kolloid-Z., 138, 6 (1954).

13. R. I. Tanner, R. E. Nickell, and R.W. Bilger, Comp. Meth. Appl. Mech. Engr., 6, 155 (1975).

14. M. J. Crochet, A. R. Davies, and K. Walters, Numerical Simulation of NonNewtonian Flow, Elsevier, Amsterdam, 1984.

Received August 15, 1988

Accepted February 10, 1989 